\title{
Tetrabromo hydrogenated cardanol: efficient and renewable brominating agent
}

Orazio A. Attanasi, ${ }^{\dagger} *$ Stefano Berretta, ${ }^{\dagger}$ Gianfranco Favi, ${ }^{\dagger}$ Paolino Filippone, ${ }^{\dagger} * *$ Giuseppe Mele, ${ }^{\ddagger}$ Giada Moscatelli, ${ }^{\dagger}$ and Raffaele Saladino ${ }^{\S}$

Centro di Sudio delle Sostanze Organiche di Origine Naturale, Università di Urbino "Carlo Bo", Via Sasso 75, 61029 Urbino, Italy, Dipartimento di Ingegneria dell'Innovazione, Università di Lecce, Via Arnesano, 73100 Lecce, Italy, and Dipartimento di Agrobiologia ed Agrochimica, Università della Tuscia, Via S. Camillo de Lellis, 01100 Viterbo, Italy

attanasi@uniurb.it; filippone@uniurb.it

\section{Supporting Information}

\section{List of Contents}

1. Spectra $\left({ }^{1} \mathrm{H}\right.$ and ${ }^{13} \mathrm{C}$ NMR) of 2,4,6-tribromo-3- $n$-pentadecylphenol and

2. ${ }^{1} \mathrm{H}$ NMR spectral data.

S3 - S4

3. ${ }^{1} \mathrm{H}$, Spectra of all compounds

$\mathrm{S} 5-\mathrm{S} 12$

4. References 
Preparation of 2,4,6-tribromo-3-n-pentadecylphenol (2): To a solution of 3-n-pentadecylphenol (16.4 mmol) dissolved in carbon tetrachloride $(30 \mathrm{~mL})$ was added dropwise a solution $1 \mathrm{M}$ of bromine in acetic acid $(62.5 \mathrm{mmol}, 20 \mathrm{~mL})$ and the mixture was magnetically stirred at room temperature for $1 \mathrm{~h}$. Then it was allowed under reflux until the disappearance of the red colour of bromine $(3-4 \mathrm{~h})$. The crude mixture was washed with water $(50 \mathrm{~mL})$, the organic layer was dried over anhydrous sodium sulphate, concentrated under reduced pressure and filtered affording 2,4,6tribromo-3-n-pentadecylphenol.

2,4,6-Tribromo-3-n-pentadecylphenol (2): $62 \%$ yield; mp 52-54 ${ }^{\circ} \mathrm{C} .{ }^{1} \mathrm{H}$ NMR $(400 \mathrm{MHz}$, $\left.\mathrm{CDCl}_{3}\right): \delta 0.88(\mathrm{t}, J=6.8 \mathrm{~Hz}, 3 \mathrm{H}), 1.26(\mathrm{~s}, 21 \mathrm{H}), 1.50(\mathrm{~m}, 5 \mathrm{H}), 2.91(\mathrm{t}, J=8.0 \mathrm{~Hz}, 2 \mathrm{H}), 5.96(\mathrm{~s}$, 1H), $7.66(\mathrm{~s}, 1 \mathrm{H}) .{ }^{13} \mathrm{C} \mathrm{NMR}\left(100 \mathrm{MHz}, \mathrm{CDCl}_{3}\right): \delta 14.1,22.7,27.9,29.2,29.4,29.7,31.9,37.3$, 106.9, 112.8, 114.7, 134.5, 141.8, 149.0. IR (nujol): 3506, 1471, 1441, 1381, 1181, 861, 784, 723, $671 \mathrm{~cm}^{-1}$. EIMS m/z (\%) $544\left(\mathrm{M}^{+}, 4\right), 542\left(\mathrm{M}^{+}, 23\right), 540\left(\mathrm{M}^{+}, 23\right), 538\left(\mathrm{M}^{+}, 4\right), 500\left(\mathrm{M}^{+}, 16\right), 498$ (68), 496 (95), 494 (45), 454 (7), 452 (15), 450 (10), 410 (33), 408 (95), 406 (100). Anal.Calcd for $\mathrm{C}_{21} \mathrm{H}_{33} \mathrm{Br}_{3} \mathrm{O}$ : C, 46.60; H, 6.15; Br, 44.29. Found: 46.44; H, 6.02; Br, 44.45.

Preparation of 2,4,4,6-tetrabromo-3-n-pentadecylcyclohexa-2,5-dienone (3) (TBPCO): To a solution of 2,4,6-tribromo-3- $n$-pentadecylphenol ( $2 \mathrm{mmol})$ dissolved in carbon tetrachloride (20 $\mathrm{mL})$ a solution $1 \mathrm{M}$ of bromine $(2.2 \mathrm{mmol})$ in the same solvent $(3 \mathrm{~mL})$ was added. Then a.catalytic amount of $\mathrm{ZnCl}_{2}(20 \%)$ was added and the mixture was magnetically stirred for $5 \mathrm{~min}$. The crude mixture was washed with a satured solution of $\mathrm{Na}_{2} \mathrm{CO}_{3}(20 \mathrm{~mL})$ and the organic layer was dried over anhydrous sodium sulphate and evaporated under reduced pressure. 2,4,4,6-Tetrabromo-3-npentadecylcyclohexa-2,5-dienone was crystallized from $\mathrm{CH}_{2} \mathrm{Cl}_{2}-\mathrm{CH}_{3} \mathrm{OH}$.

2,4,4,6-Tetrabromo-3-n-pentadecylcyclohexa-2,5-dienone (3): $90 \%$ yield; mp 58-60 ${ }^{\circ} \mathrm{C} .{ }^{1} \mathrm{H}$ NMR (400 MHz, $\left.\mathrm{CDCl}_{3}\right): \delta 0.88(\mathrm{t}, J=6.4 \mathrm{~Hz}, 3 \mathrm{H}), 1.26(\mathrm{~s}, 22 \mathrm{H}), 1.52(\mathrm{~m}, 2 \mathrm{H}), 1.92(\mathrm{~m}, 2 \mathrm{H}), 2.79$ $(\mathrm{m}, 2 \mathrm{H}) 7.82(\mathrm{~s}, 1 \mathrm{H}) .{ }^{13} \mathrm{C} \mathrm{NMR}\left(100 \mathrm{MHz}, \mathrm{CDCl}_{3}\right): \delta 14.1,22.7,28.1,29.1,29.4,29.5,29.6,29.7$, 30.1, 31.9, 38.3, 51.8, 118.4, 122.3, 147.6, 147.7, 157.2, 170.4. IR (nujol): 1679, 1577, 1465, 1190, 892, 705, 678, $629 \mathrm{~cm}^{-1}$. EIMS m/z (\%) $624\left(\mathrm{M}^{+}, 4\right), 622\left(\mathrm{M}^{+}, 13\right), 620\left(\mathrm{M}^{+}, 21\right), 618\left(\mathrm{M}^{+}, 13\right), 616$ 
$\left(\mathrm{M}^{+}, 4\right), 544$ (2), 542 (7), 540 (7), 538 (2), 471 (1), 425 (28), 423 (43), 421 (28), 343 (100).

Anal.Calcd for $\mathrm{C}_{21} \mathrm{H}_{32} \mathrm{Br}_{4} \mathrm{O}$ : C, 40.68; H, 5.20; Br, 51.54. Found: 40.46; H, 5.39; Br, 51.38.

General Experimental Procedure for Bromination of Organic Substrates: To a stirred solution of organic substrate $(0.5 \mathrm{mmol})$ dissolved in the appropriate solvent $(10 \mathrm{~mL})$ (Table 1$)$ was added in small portions 2,4,4,6-tetrabromo-3- $n$-pentadecyl-2,5-cyclohexadienone (0.55 mmol). The reaction mixture was magnetically stirred until the disappearance of the starting organic substrate (monitored by TLC). After completion of the reaction, the reaction mixture was concentrated under reduced pressure and was purified by crystallization of 2,4,6-tribromo-3- $n$-pentadecylphenol from $\mathrm{CH}_{3} \mathrm{OH}$. Brominated organic substrate was obtained in satisfactory yield and purity.

All the bromo derivatives are known compounds reported in the literature.

$N$-(4-Bromophenyl)- $N, N$-diethylamine : ${ }^{1} \mathrm{H}-\mathrm{NMR}$ spectrum is in accordance with that reported in the literature. ${ }^{1}{ }^{1} \mathrm{H}$ NMR (400 MHz, $\left.\mathrm{CDCl}_{3}\right): \delta 1.15(\mathrm{t}, J=7.2 \mathrm{~Hz}, 6 \mathrm{H}), 3.32(\mathrm{q}, J=7.2 \mathrm{~Hz}, 4 \mathrm{H})$, $6.56(\mathrm{~d}, J=8.8 \mathrm{~Hz}, 2 \mathrm{H}), 7.27(\mathrm{~d}, J=8.8 \mathrm{~Hz}, 2 \mathrm{H})$.

2-Bromo-4-methoxyaniline : ${ }^{1} \mathrm{H}-\mathrm{NMR}$ spectrum is in accordance with that reported in the literature. ${ }^{2}{ }^{1} \mathrm{H}$ NMR $\left(400 \mathrm{MHz}, \mathrm{CDCl}_{3}\right): \delta 3.73(\mathrm{~s}, 3 \mathrm{H}), 4.82(\mathrm{brs}, 2 \mathrm{H}), 6.73\left(\mathrm{dd}, J_{l}=2.8 \mathrm{~Hz}, J_{2}=\right.$ $8.8 \mathrm{~Hz}, 1 \mathrm{H}), 6.78(\mathrm{~d}, J=8.8 \mathrm{~Hz}, 1 \mathrm{H}), 7.01(\mathrm{~d}, J=2.8 \mathrm{~Hz}, 1 \mathrm{H})$.

2-Bromo-4-tert-butylphenol : ${ }^{1} \mathrm{H}-\mathrm{NMR}$ spectrum is in accordance with that reported in the literature. ${ }^{3}{ }^{1} \mathrm{H} \mathrm{NMR}\left(400 \mathrm{MHz}, \mathrm{CDCl}_{3}\right): \delta 1.28(\mathrm{~s}, 9 \mathrm{H}), 5.35(\mathrm{~s}, 1 \mathrm{H}), 6.95(\mathrm{~d}, J=8.4 \mathrm{~Hz}, 2 \mathrm{H}), 7.23$ $\left(\mathrm{dd}, J_{l}=2.4 \mathrm{~Hz}, J_{2}=8.4 \mathrm{~Hz}, 1 \mathrm{H}\right), 7.44(\mathrm{~d}, J=2.4 \mathrm{~Hz}, 1 \mathrm{H})$.

4-Bromo-2,6-dimethylphenol : ${ }^{1} \mathrm{H}-\mathrm{NMR}$ spectrum is in accordance with that reported in the literature. ${ }^{4}{ }^{1} \mathrm{H}$ NMR $\left(400 \mathrm{MHz}, \mathrm{CDCl}_{3}\right): \delta 2.21$ (s, 6H), 4.59 (brs, 1H), 7.09 (s, 2H).

1-Bromo-2-naphthol : ${ }^{1} \mathrm{H}-\mathrm{NMR}$ spectrum is in accordance with ${ }^{1} \mathrm{H}-\mathrm{NMR}$ spectrum of material purchased from Aldrich Chemical Co., Inc. and with that reported in the literature. ${ }^{5}{ }^{1} \mathrm{H}$ NMR (400 $\left.\mathrm{MHz}_{\mathrm{CDCl}}\right): \delta 5.96(\mathrm{~s}, 1 \mathrm{H}), 7.27\left(\mathrm{dd}, J_{l}=0.8 \mathrm{~Hz}, J_{2}=8.4 \mathrm{~Hz}, 1 \mathrm{H}\right), 7.39\left(\mathrm{dt}, J_{l}=0.8 \mathrm{~Hz}, J_{2}=8.4\right.$ 
$\mathrm{Hz}, 1 \mathrm{H}), 7.57\left(\mathrm{dt}, J_{l}=0.8 \mathrm{~Hz}, J_{2}=8.4 \mathrm{~Hz}, 1 \mathrm{H}\right), 7.73(\mathrm{~d}, J=8.4 \mathrm{~Hz}, 1 \mathrm{H}), 7.77(\mathrm{~d}, J=8.4 \mathrm{~Hz}, 1 \mathrm{H})$, $8.03(\mathrm{~d}, J=8.4 \mathrm{~Hz}, 1 \mathrm{H})$.

2-Bromo-1-phenyl-1,3-butanedione : ${ }^{1} \mathrm{H}-\mathrm{NMR}$ spectrum is in accordance with that reported in the literature. ${ }^{6}{ }^{1} \mathrm{H} \mathrm{NMR}\left(400 \mathrm{MHz}, \mathrm{CDCl}_{3}\right): \delta 2.43(\mathrm{~s}, 3 \mathrm{H}), 5.63(\mathrm{~s}, 1 \mathrm{H}), 7.48(\mathrm{~m}, 2 \mathrm{H}), 7.62(\mathrm{~m}, 1 \mathrm{H})$, $7.96(\mathrm{~m}, 2 \mathrm{H})$.

Ethyl 2-bromo-3-oxo-3-phenylpropanoate : ${ }^{1} \mathrm{H}-\mathrm{NMR}$ spectrum is in accordance with that reported in the literature. ${ }^{7} \mathrm{H}$ NMR $\left(400 \mathrm{MHz}, \mathrm{CDCl}_{3}\right): \delta 1.22(\mathrm{t}, 3 \mathrm{H}), 4.28(\mathrm{q}, 2 \mathrm{H}), 5.64(\mathrm{~s}, 1 \mathrm{H})$ $7.50(\mathrm{~m}, 2 \mathrm{H}) 7.62(\mathrm{~m}, 1 \mathrm{H}) 8.00(\mathrm{~m}, 2 \mathrm{H})$.

N1-(4-Methoxyphenyl)-2-bromo 3-oxobutanamide : ${ }^{1} \mathrm{H}-\mathrm{NMR}$ spectrum is in accordance with that reported in the literature. ${ }^{8}{ }^{1} \mathrm{H}$ NMR $\left(400 \mathrm{MHz}, \mathrm{CDCl}_{3}\right): \delta 2.48(\mathrm{~s}, 3 \mathrm{H}), 3.80(\mathrm{~s}, 3 \mathrm{H}), 4.87$ (s, $1 \mathrm{H}), 6.88(\mathrm{~d}, J=8.8 \mathrm{~Hz}, 1 \mathrm{H}), 7.43(\mathrm{~d}, J=8.8 \mathrm{~Hz}, 1 \mathrm{H}), 8.28(\mathrm{~s}, 1 \mathrm{H})$.

1-Bromo-2-oxo-2-phenylethyl cyanide : ${ }^{1} \mathrm{H}-\mathrm{NMR}$ spectrum is in accordance with that reported in the literature. ${ }^{9}{ }^{1} \mathrm{H} \mathrm{NMR}\left(400 \mathrm{MHz}, \mathrm{CDCl}_{3}\right): \delta 5.63(\mathrm{~s}, 1 \mathrm{H}), 7.54(\mathrm{t}, J=7.6 \mathrm{~Hz}, 2 \mathrm{H}), 7.65(\mathrm{t}, J=7.6$ $\mathrm{Hz}, 1 \mathrm{H}), 7.99$ (d, $J=7.6 \mathrm{~Hz}, 2 \mathrm{H})$.

Ethyl 3-[2-(aminocarbonyl)hydrazono]-2-bromobutanoate: ${ }^{1} \mathrm{H}-\mathrm{NMR}$ spectrum is in accordance with that reported in the literature. ${ }^{10}{ }^{1} \mathrm{H}$ NMR (400 MHz, $\left.\mathrm{CDCl}_{3}\right): \delta 1.30(\mathrm{t}, J=7.2 \mathrm{~Hz}, 3 \mathrm{H}), 2.07$ (s, 3H), $4.25(\mathrm{q}, J=7.2 \mathrm{~Hz}, 2 \mathrm{H}), 5.02(\mathrm{~s}, 1 \mathrm{H}), 6.10$ (brs, 2H), 8.84 (s, 1H).

1-Bromo-1-(4-nitrophenyl)acetone : ${ }^{1} \mathrm{H}-\mathrm{NMR}$ spectrum is in accordance with that reported in the literature. ${ }^{11}{ }^{1} \mathrm{H}$ NMR $\left(400 \mathrm{MHz}, \mathrm{CDCl}_{3}\right): \delta 2.40(\mathrm{~s}, 3 \mathrm{H}), 5.45(\mathrm{~s}, 1 \mathrm{H}), 7.62(\mathrm{~d}, J=8.8 \mathrm{~Hz}, 2 \mathrm{H}), 8.23$ $(\mathrm{d}, J=8.8 \mathrm{~Hz}, 2 \mathrm{H})$.

2-Bromo-cyclohexanone : ${ }^{1} \mathrm{H}-\mathrm{NMR}$ spectrum is in accordance with that reported in the literature. ${ }^{4}$ ${ }^{1} \mathrm{H}$ NMR $\left(400 \mathrm{MHz}, \mathrm{CDCl}_{3}\right): \delta 1.72(\mathrm{~m}, 1 \mathrm{H}), 1.81(\mathrm{~m}, 1 \mathrm{H}), 1.94(\mathrm{~m}, 1 \mathrm{H}), 2.03(\mathrm{~m}, 1 \mathrm{H}), 2.22(\mathrm{~m}$, 1H), 2.35 (m, 2H), $2.98(\mathrm{~m}, 1 \mathrm{H}), 4.44(\mathrm{~m}, 1 \mathrm{H})$. 

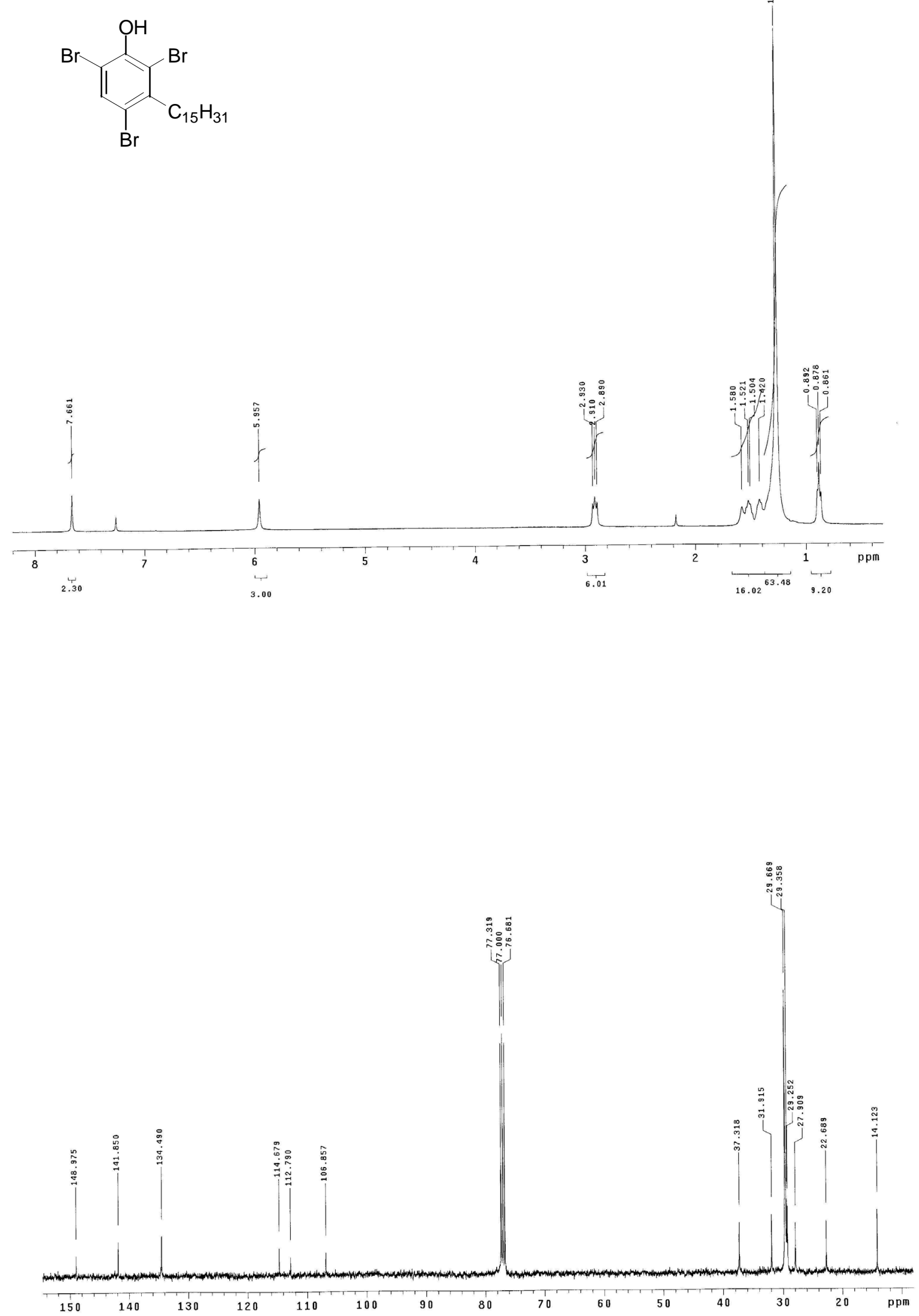

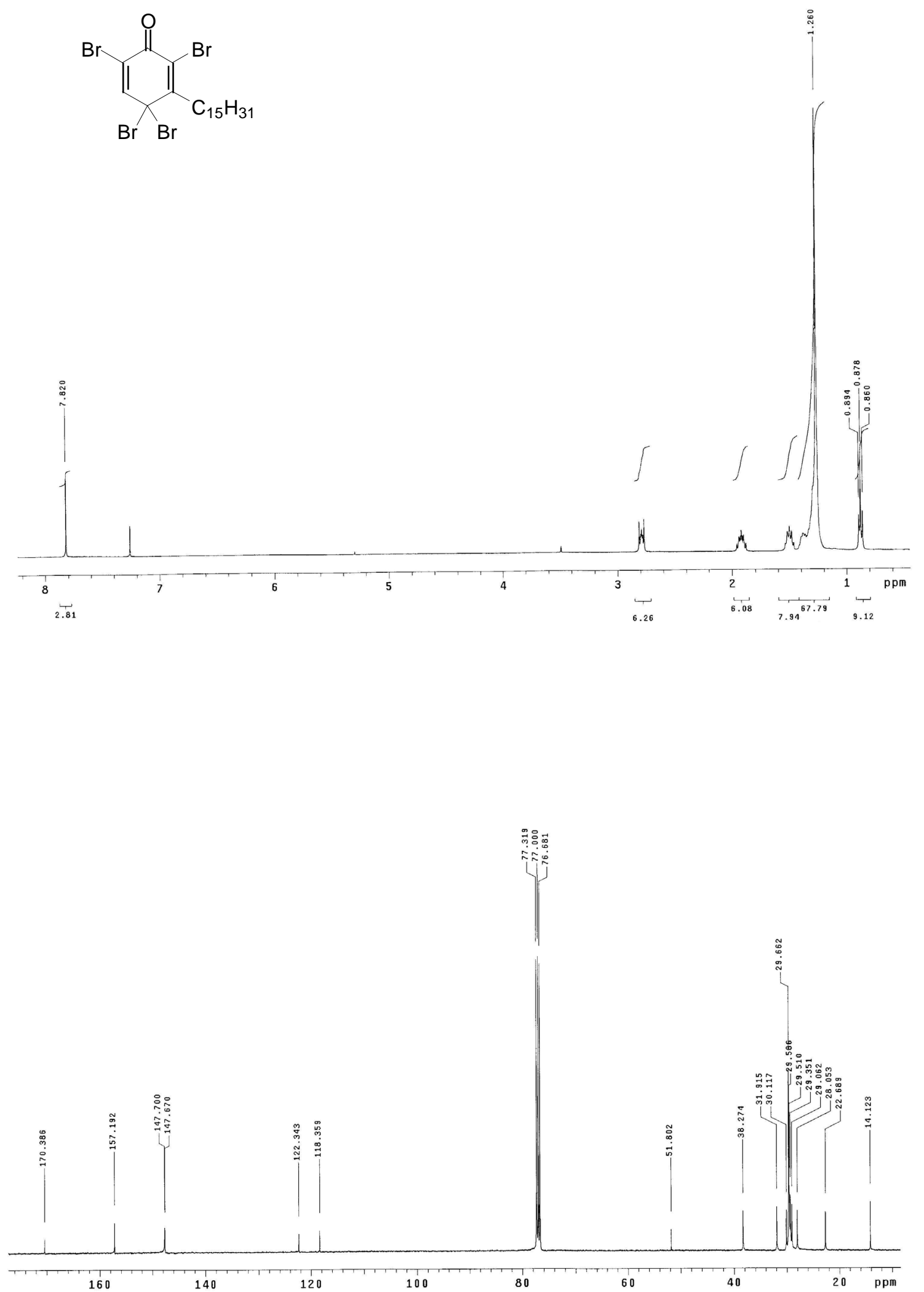

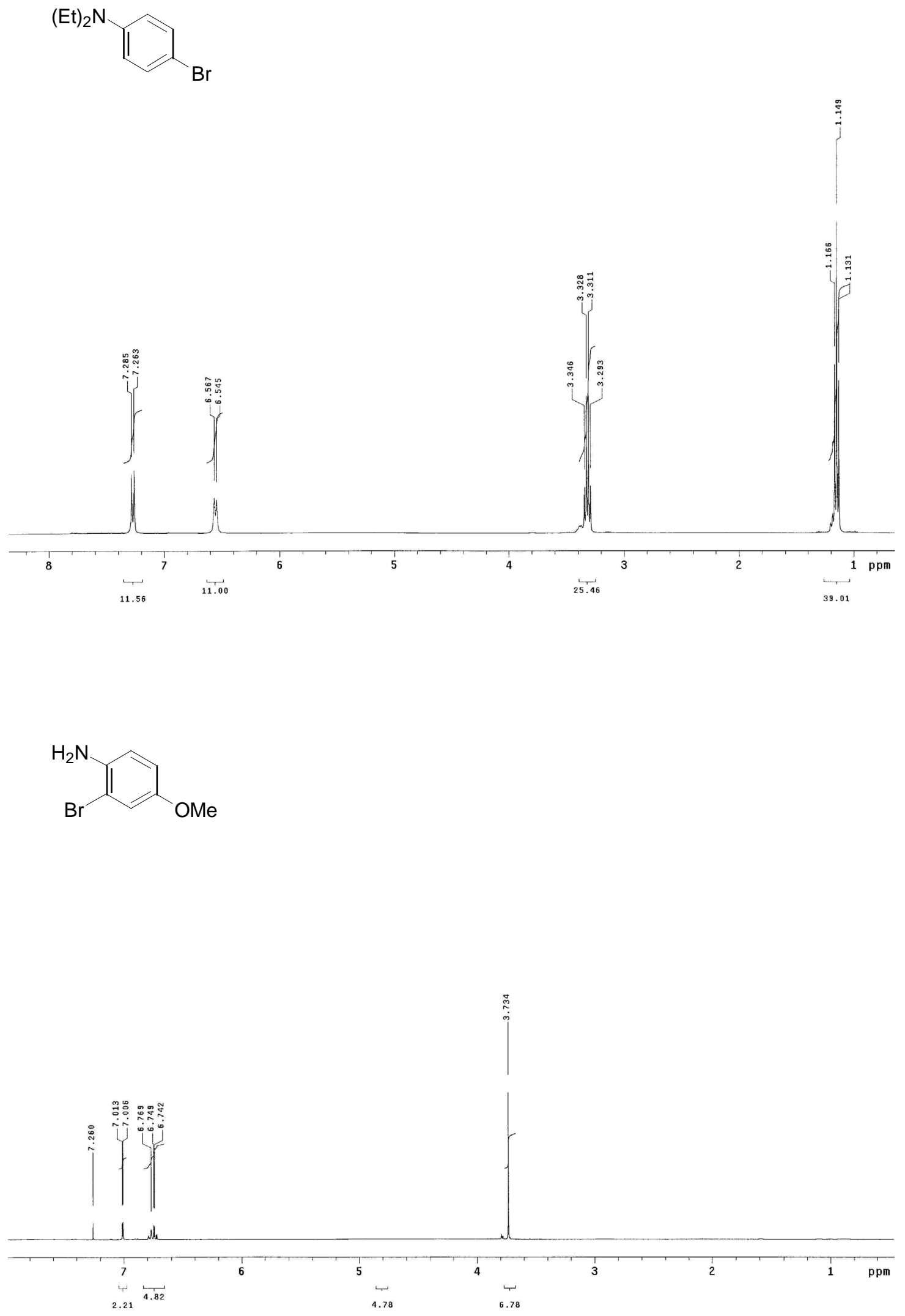

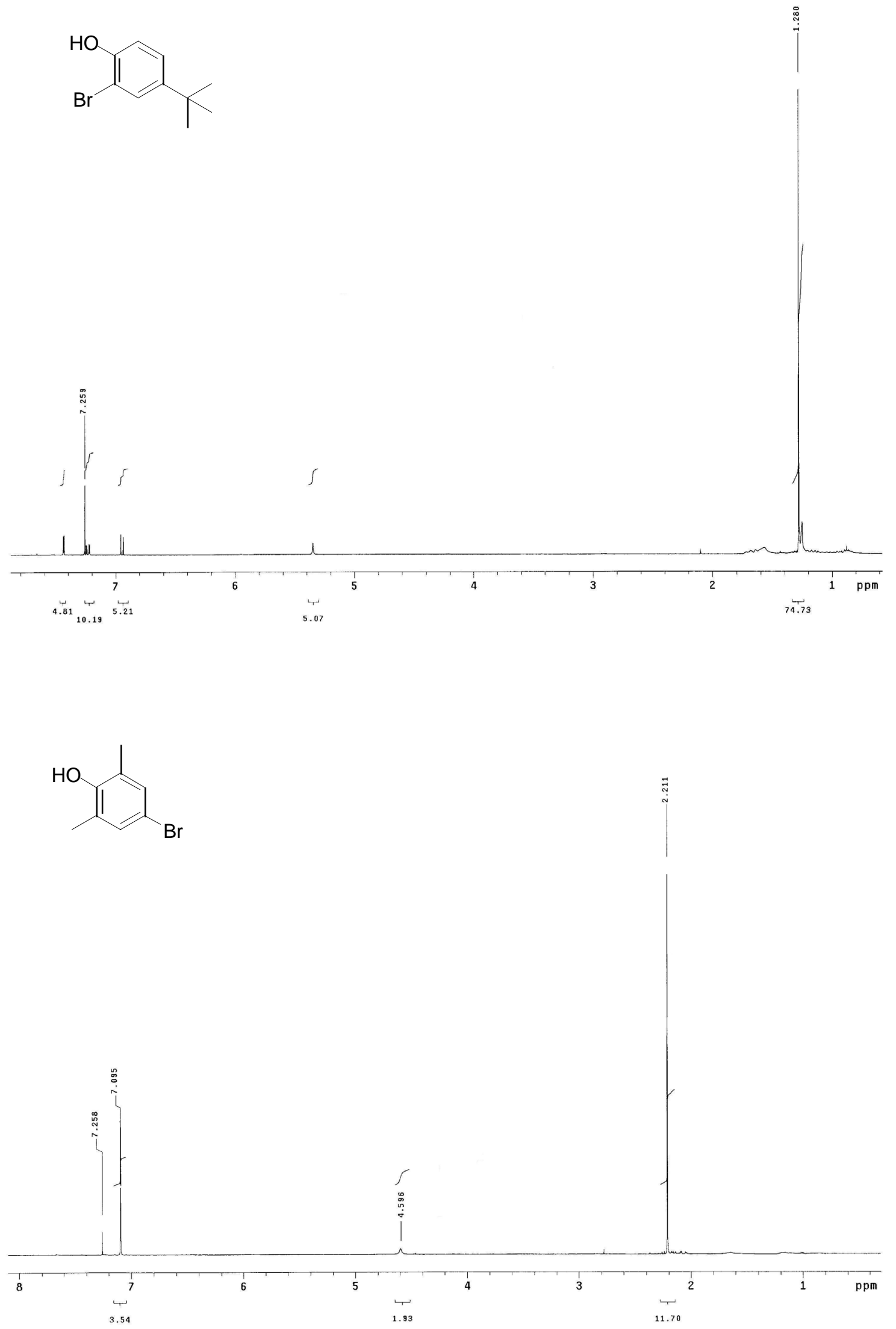
<smiles>Oc1ccc2ccccc2c1Br</smiles>
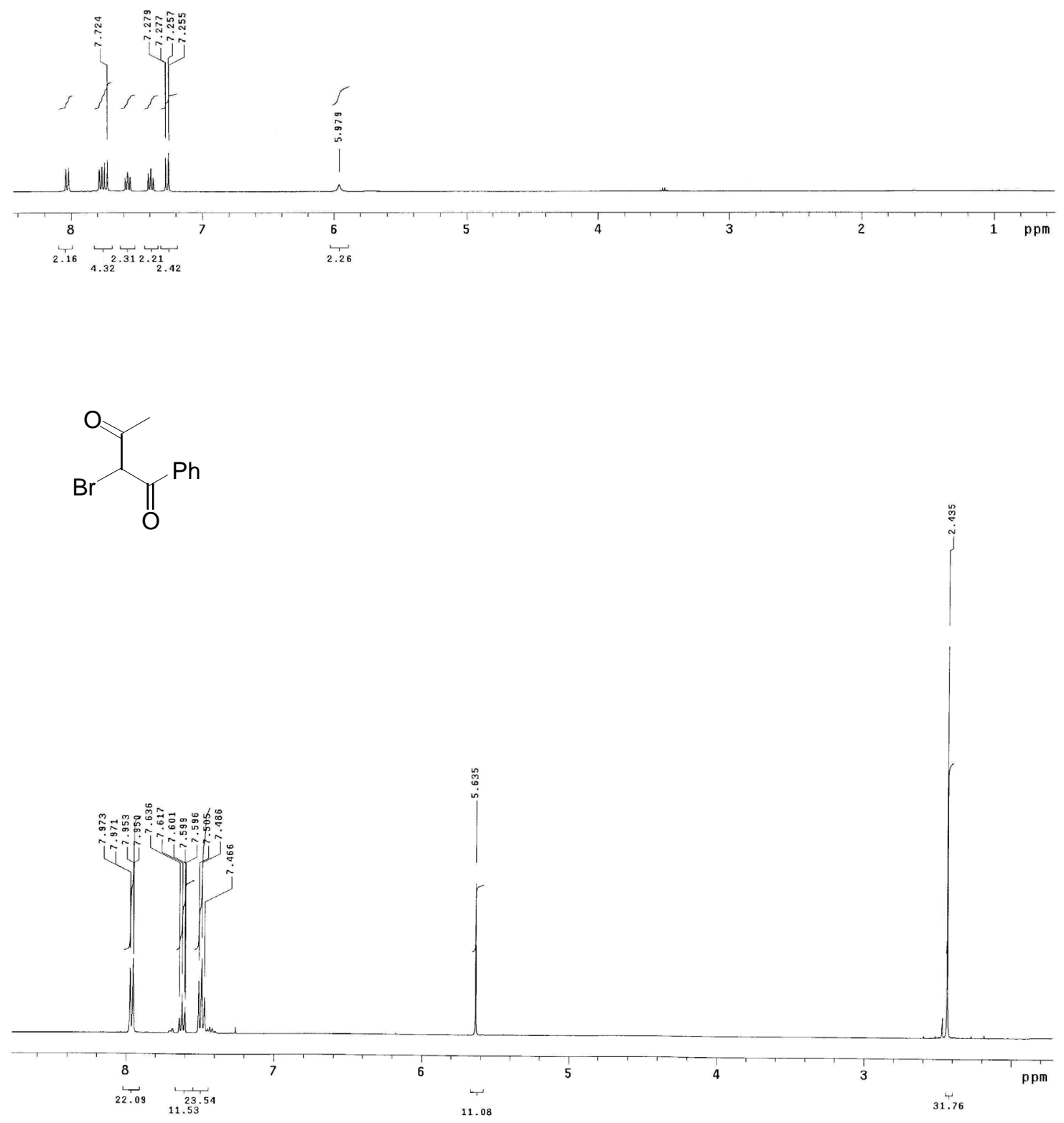

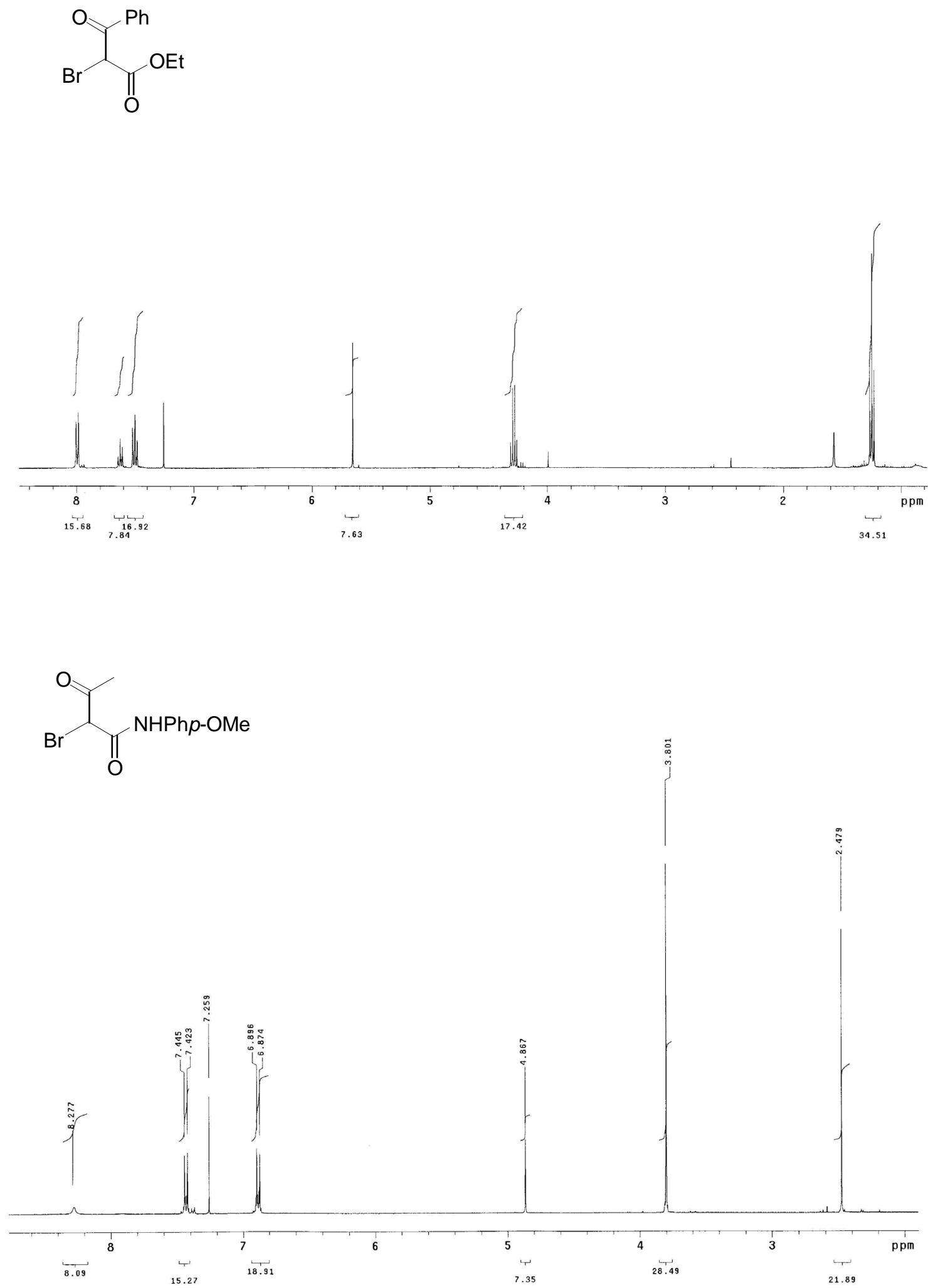

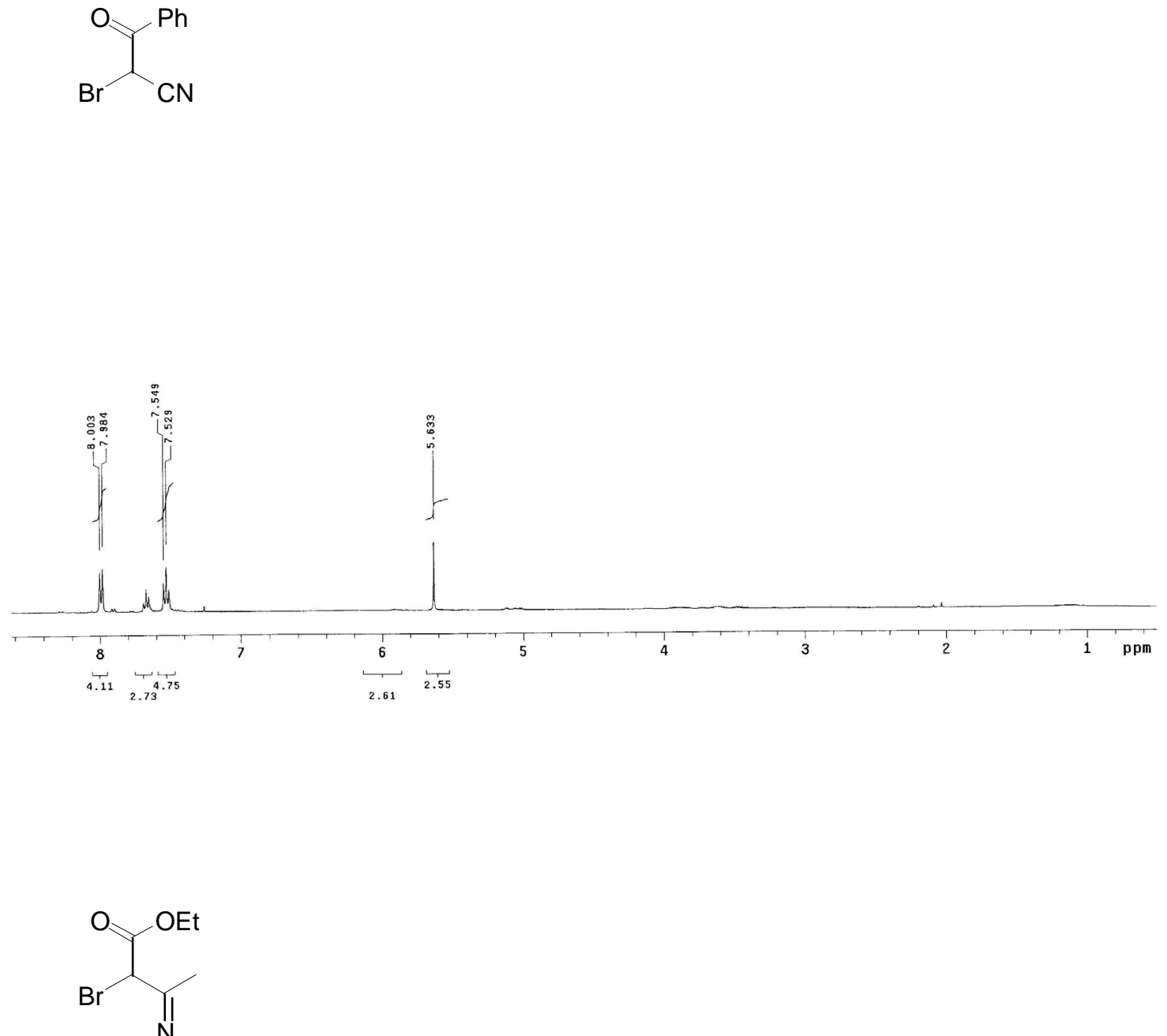

$\mathrm{NHCONH}_{2}$

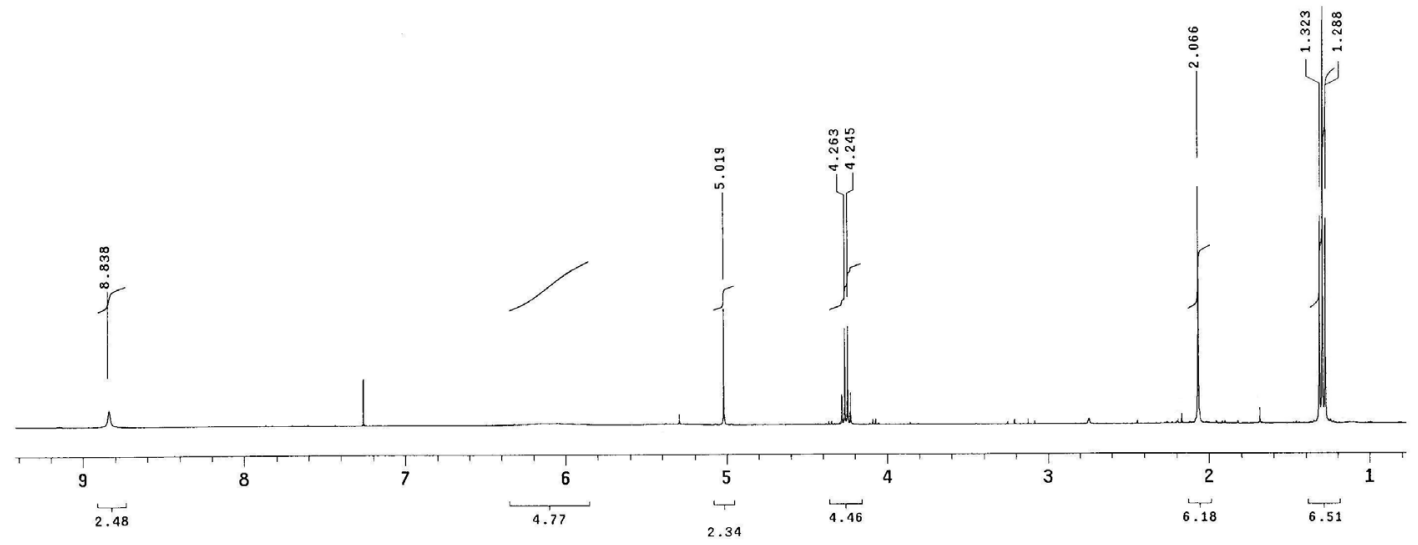



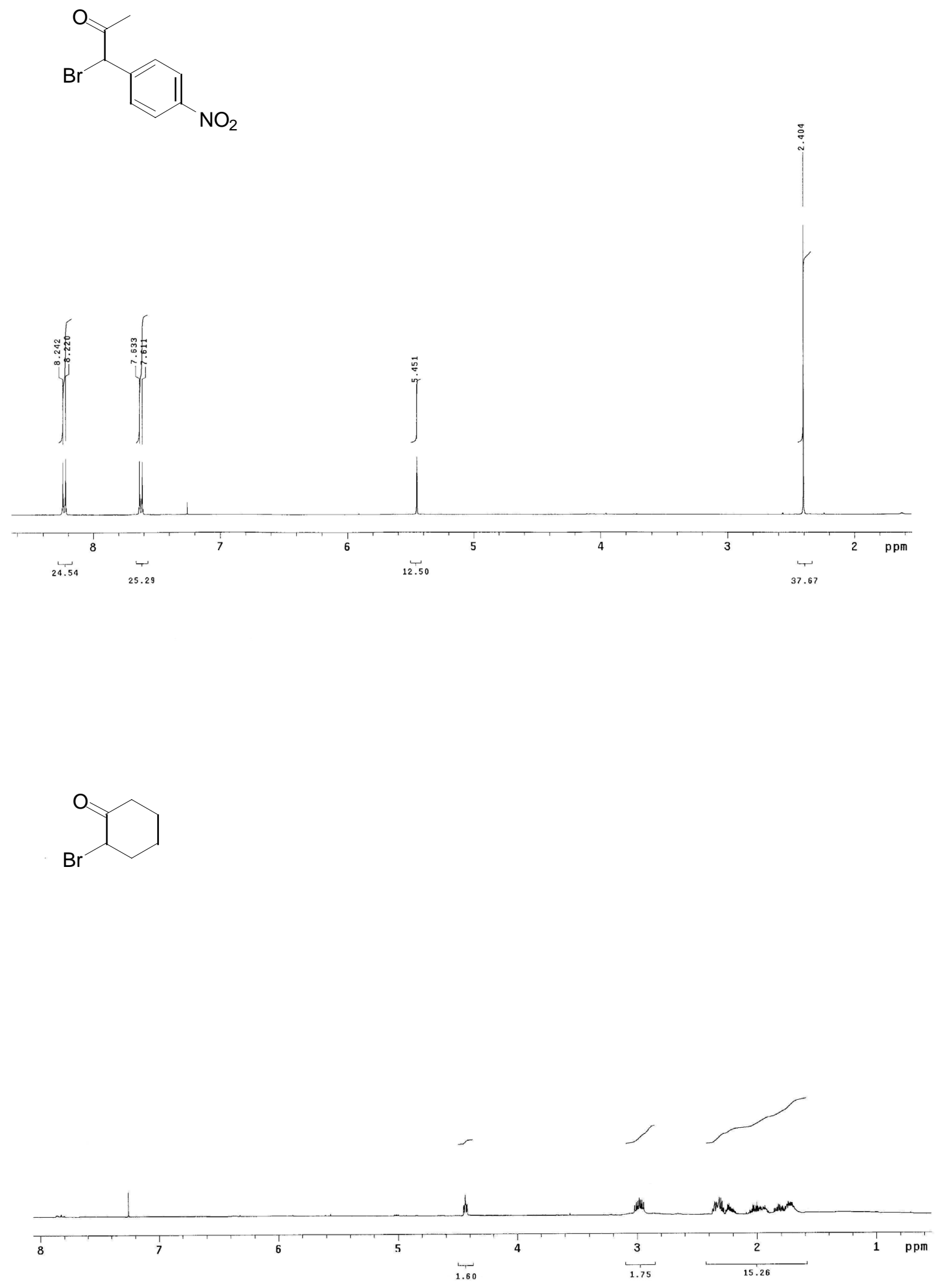


\section{References:}

(1) Cerichelli, G.; Mancini, G.; Luchetti, L. Tetrahedron 2001, 50, 3797.

(2) Chrétien, J.-M.; Zammattio, F.; Le Grognec, E.; Paris, M.; Cahingt, B.; Montavan, G.; Quintard, J.-P. J. Org. Chem. 2005, 70, 2870.

(3) Cai, L.; Mahmoud, H.; Han, Y. J. Tetrahedron: Asymmetry 1999, 411.

(4) Kavala, V.; Naik, S.; Patel, B. K. J. Org. Chem. 2005, 70, 426.

(5) Bora, U.; Bose, G.; Chaudhuri, M. K.; Dhar, S. S.; Gopinath, R.; Khan, A. T.; Patel, B. K. Org. Lett. 2000, 2, 247.

(6) Choi, H. Y.; Chi, D. Y. Org. Lett. 2003, 5, 411.

(7) Yang, D.; Yan, Y.-L.; Lui, B. J. Org. Chem. 2002, 67, 7429.

(8) Arrieta, A.; Ganboa, I.; Palomo, C. Synth. Commun. 1984, 14, 939.

(9) Batanero, B.; Vago, M.; Barba, F. Heterocycles. 2000, 53, 1337.

(10) Attanasi, O. A.; Filippone, P.; Mei A.; Santeusanio, S. Synthesis 1984, 873.

(11) Takano, S.; Imaizumi, H.; Kajita, T.; Takashima, K.; Takezawa, K.; Yotsutsuji, M.; Yasuda, T.; Yotsutsuji, A.; Sakai, H.; Saikawa, I. Jpn. Patent 62178590, 1987. 